\title{
Fault-Tolerant Energy-Efficient Priority-Based Routing Scheme for the Multisink Healthcare Sensor Networks
}

\author{
Ather Saeed, Andrew Stranieri, and Richard Dazeley \\ Centre for Informatics and Applied Optimization, University of Ballarat and Melbourne Institute of Technology, Ballarat, \\ VIC 3350, Australia \\ Correspondence should be addressed to Ather Saeed, asaeed@mit.edu.au
}

Received 11 January 2012; Accepted 23 February 2012

Academic Editor: F. Viani

Copyright (๑) 2012 Ather Saeed et al. This is an open access article distributed under the Creative Commons Attribution License, which permits unrestricted use, distribution, and reproduction in any medium, provided the original work is properly cited.

\begin{abstract}
Wireless sensor networks (WSNs) are widely used in battle fields, logistic applications, healthcare, habitat monitoring, environmental monitoring, home security, and variety of other areas. The existing routing algorithms focus on the delivery of data packets to the sink using the shortest path; however, calculating the shortest path is not a cost-effective solution while disseminating datasets of interest to the nearest sink node. The approach presented in this paper extends the existing PBR (priority-based routing) protocol by providing a new fault-tolerant multipath priority-based routing (FT-MPPBR) scheme, which not only balances the energy consumption while selecting multiple paths but also balances the workload of the node closest to the sink. The nodes closer to the sink dissipate more energy and can become the source of a communication bottleneck. Simulation results for the proposed routing scheme are encouraging and clearly show that the FT-MPPBR has outperformed the existing PBR schemes in terms of prolonging the network lifetime and reliability. In healthcare sensor networks, timely dissemination of datasets is critical for the well-being of a patient. This research further extends the PBR architecture for supporting computational intensive analysis by transferring datasets of interest to the sensor grid node for improved communication and better throughput.
\end{abstract}

\section{Introduction}

Fault tolerance is particularly important in sensor networks especially when such devices are attached to the patients with serious medical conditions and monitoring of vital signs is crucial for their survival.

The existing algorithms [1-7] for disseminating datasets of interests have widely ignored fault tolerance while communicating datasets to the sink node. In-network processing of datasets received from the sensor nodes attached to the patients with cardiovascular problems, Parkinson disease, epilepsy, high blood pressure, carbon-dioxide concentration, oxygen saturation in the blood, and ECG needs to be efficiently monitored for the future forecasting. Any sudden changes will be communicated to the relevant GP soon after the threshold values trigger alarms.

The presence of fault at a particular node can easily compromise the quality of information communicated through a malfunctioning node with relevant information which could be life threatening. A fault-tolerant solution for achieving the desired functionality in the presence of fault is important.

The issue of fault tolerance has widely been ignored in [3-5], which is crucial for the energy-efficient data acquisition and dissemination in sensor networks.

The multipath routing algorithms discussed in [5-7] also have a problem of receiving similar packets from various paths. If all such packets are routed to the sink then network latency and communication overheads will increase significantly.

In wireless sensor networks major challenge is to prolong the battery life by reducing communication overheads. This paper focuses on the fault-tolerant aspects of information dissemination to the sensor nodes so that reliability and quality of service can be improved once the data is transferred to the sink node.

Efficient information retrieval is a challenge in such environments because of high energy consumption, low processing capacity, and low memory problems in such devices. 
Datasets of interest are available once the query is propagated through the sink node; the challenge is to look at the responsible node that has an answer to that query and also to find the distance of that node from the sink. Nodes that are far from the sink consume more energy while disseminating information. In other words the battery will be depleted quickly, which will result in the degradation of the whole network.

This research looks further at how the updates of interest can be routed to the sink node soon after they are available.

Healthcare is a sensitive domain; if the information is not communicated at the right time to the right source, then it could be life threatening for the patient. Most wireless devices work on hard threshold values, which means if blood pressure is already at 199 and threshold value is 200 then such crucial stage will not be monitored and picked by the device attached to the patient.

Existing algorithms and proposed methodologies discussed in [1-5, 7-15] do not provide a fault-tolerant solution for the dissemination and routing of datasets and the fault-tolerant aspects of communication overheads are also ignored. Such issues will be addressed in this research, which not only looks at the aggregation function in the context of fault tolerance but also look at the duplicate sensitive queries for reducing communication overheads.

A better approach is required to overcome problems such as information availability and accessibility in such environments while communicating datasets of interest through critical paths.

This research extends the model discussed in [2, 3] and proposes a new fault-tolerant multipath priority-based routing scheme (FT-MPPBR) that also takes into account malfunctioning of the sensor node responsible for communication in order to achieve the desired functionality while disseminating datasets of interests through critical paths.

The main goal is to investigate the efficiency of proposed approach while disseminating datasets through multipath and how effectively it balances the energy consumption before routing datasets to the nearest sink for prolonging the network life.

\section{Related Work}

Wireless sensor networks (WSNs) operate in a complex environment when it comes to the retrieval of information from nodes that are interconnected using a certain network topology. Information retrieval (IR) from such nodes poses a great challenge as the information of interest is available at the node that is located far from the base station or sink. The second biggest challenge is to efficiently maintain balance between energy consumption and the routing technique used for the dissemination of information to the sink node.

Wireless sensor devices have low battery, limited lifetime, low processing power, and limited memory. They operate in a highly sensitive environment, because of the abovementioned problems, a node responsible for routing the result of query propagated through the sink might be out of battery or suffering from the data loss due to problem with the neighboring nodes. Answering such an enormous amount of queries is a resource and computational intensive task.

Information availability also poses a challenge when it comes to information retrieval from the relevant nodes of interest, limited battery, and quality of sensor nodes that are interconnected are always the main concern during the data dissemination phase.

Fault tolerance is widely ignored by the proposed routing techniques and algorithms mentioned in [1-7, 9-12] for the retrieval of information from wireless sensor nodes.

Fault tolerance is the ability to provide desired functionality despite the presence of faults. Most of the existing techniques mentioned above did not address the issue of fault tolerance properly but did mention about the need for a better approach for data acquisition and dissemination through critical paths.

Data aggregation and energy balanced routing are discussed in [10]; reference [11] addresses the issue of extending the battery life up to some extent by reducing communication overheads. Issues of fault tolerance were totally ignored while measuring effectiveness of their proposed approach.

Data aggregation $[1-3,10,11,16]$ is a process of gathering useful and relevant information that is scattered around various distributed and decentralized nodes connected for exchanging information of interest to the base station without losing data packets.

It is also important to discuss the techniques and protocols designed for aggregation of data from sensor nodes based on the network architecture. Protocols that work on the flat networks use data centric scheme, sink usually advertises the query by flooding, and each node has exactly the same level of energy in terms of battery and resource consumption.

Most of these protocols have benefits and limitations which will be discussed in detail. Sensor Protocol for Information via Negotiation (SPIN) [17] uses push diffusion scheme for negotiation and resource adaptation. Directed diffusion [5] is a two-phase pull diffusion scheme, if there is a match in interest in terms of data generated then the gradient will be set up to determine the direction and the flow of information to the sink.

Similarly, hierarchical networks are capable of providing the desired functionality by looking at energy consumption and scalability. Protocols that work at hierarchical level are LEACH, HEED, PEGASIS, EADAT, and PEDAP-PA and most of them provide an efficient scheme for the propagation and dissemination of information to the sink node for the in-network processing of information, which can be used for future forecasting but widely ignored. Malfunctioning of node should be taken into account while communicating datasets to the nearest sink.

Achieving fault tolerance in wireless sensor networks is challenging because of the limited processing power and energy consumption problem in such devices. An enormous amount of work has been done in this particular area but scalability and energy consumption of network was not properly looked at by the above-mentioned protocols, which is crucial for achieving fault tolerance. 
Faults in wireless sensor networks can occur at different levels such as, hardware, network communication, software, and application level as mentioned in [13-15]. One of the common methods used to eliminate faults is to introduce redundancy or replication of components that are prone to failure. For example, PTAS (Polynomial time approximation scheme), STP-MSP (Steinser tree problem with minimum number of Steiner points), and other, are used for detecting faults at hardware level.

Target and event detection are crucial in specific region. Value fusion and decision fusion are the two popular algorithms used for event and target detection in an Euclidian plane. Data aggregation and multipath routing protocols are used for enhancing fault tolerance in the system. These protocols typically focus on balancing energy consumption and routing scheme for the dissemination of datasets.

The proposed techniques for data aggregation, replication and routing schemes mentioned in [1-7, 9, 13-15] very briefly address the need for a new architecture for supporting in-network processing of computational intensive queries that are routed in the data intensive environment. Presence of fault due to the malfunctioning node will not only increase the network congestion but will significantly increase the network latency as well.

This research typically explores those areas which were not properly looked at in terms of fault tolerance. It also provides a solution to the content- and context-sensitive queries that are routed periodically for monitoring particular thresholds in which users are interested in.

These partial solutions $[1-7,9,13-15,18-30]$ for data aggregation, data replication, information monitoring, and data extraction address the issues and problems with wireless sensor networks up to some extent, where the major focus was to find cost-effective solution for routing datasets to the nearest sink but the issue of malfunctioning nodes while constructing a network topology is widely ignored.

Our research typically explores the issues related to data acquisition, dissemination, and malfunctioning of the sensor node for ensuring reliable delivery of datasets to the nearest sink. Below are the points that need to be taken into account for prolonging the sensor network life.

(i) Information retrieval and data extraction in wireless sensor networks is a problem $[18,19]$ because they are energy-constrained devices with low memory, limited processing power, and battery problem. Extending the lifetime of wireless sensor networks is a challenge because of the sensitive nature of the wireless devices. A priority-based routing scheme is proposed to optimize the balance between energy consumption and path selection.

(ii) Our research also provide new techniques for aggregating duplicate sensitive datasets of interest from remote locations by looking at the relevance as the main metric. Here relevance means information users are interested in. Most of the existing routing techniques $[13,16,18,31-35]$ suffer from a problem of data loss because of not adequately considering communication overheads, which can quickly deplete the batteries and increase the network latency. The problem with flooding technique is that it routes all packets to the sink node without considering the routing of same datasets over and over again, which will significantly affect the battery energy consumption, crucial for the lifetime of sensor networks; details are discussed in next section.

Research objectives and FT-MPPBR algorithm are discussed in the Section 3 in more detail, that also provide an overview of the problem domain and emphasizes on the need for a new routing scheme for ensuring fault tolerance.

Experimental analyses are discussed in Section 4, which provides comparison of proposed priority-based routing scheme with the existing multi sink and other routing algorithms.

Conclusion is discussed in Section 5, which provides the summarized overview of proposed approach and the future work in progress in context of fault tolerance.

\section{Proposed Methodology}

The proposed scheme looks at balancing the energy consumption level, while transmitting datasets of interests through the critical path. The scheme which is discussed in $[2,3]$ is extended to support multiple sinks. Path to the nearest sink is constructed from point " $P$ "; energy level of the critical path to the nearest sink is determined first before routing the datasets of interest.

A parallel aggregator is introduced near every sink so that data loss can be minimized by efficiently identifying the malfunctioning nodes. Nodes closer to the sink dissipate more energy as compare, to the other nodes to enhance the fault tolerance by efficiently routing datasets closer to a particular sink.

The parallel aggregator will only be activated in case of problem with the first aggregator so that datasets of interests can be routed through the second aggregator. Detailed analysis of the single and multipath approach is discussed in the experiment section in relation to the data loss problem.

3.1. Challenges and Issues. Understanding the topology construction in multisink sensor networks is important while disseminating datasets of interest. The following issues are important to be taken into account during the data dissemination phase.

(i) The distance of sink from the sensor node is proportional to the amount of energy consumed during each communication. In other words more energy would be consumed if the source is at a maximum distance.

(ii) To balance the energy consumption by efficiently removing the duplicate sensitive datasets so that unnecessary communication overheads can be reduced.

(iii) The aggregation scheme discussed in $[5,6]$ addresses the problem of energy consumption up to some extent but ignored the fact that sensor nodes or cluster head closer to the sink dissipate energy quickly and can break down the whole network communication. 
(iv) Another fact which is ignored in $[2,3]$ is to set the priority efficiently, in case of problem with the node closer to the sink. Routing table will be recalculated and the path closer to the sink will be computed before routing datasets through a particular path.

(v) The path with maximum energy level is selected for routing datasets and can be aggregated in the direction of the sink closer to source; priority " $P$ " of the node will be set accordingly; topology construction in the direction of available sink needs to be taken into account properly for minimizing network latency.

(vi) Our approach further extends the model discussed in $[2,3]$ by introducing fault tolerance through multipath selection. The parallel aggregators closer to each sink are introduced to minimize the network latency and reduce unnecessary communication overheads. The main challenge is to avoid break down of the whole communication in the presence of fault.

3.2. Proposed Approach. Proposed approach is shown in Figures 1 and 2 above. The model discussed in $[2,3]$ is extended to support multipath priority-based routing. A hybrid approach is proposed which not only takes energy consumption into account but also looks at the path selection before disseminating datasets of interest to the sink node. Two parallel aggregators close to each sink are introduced. The purpose is to balance the workload and energy consumption of nodes closer to each sink.

If the energy levels of aggregators $\left(A_{1}, A_{-1}\right)$ or $\left(A_{2}, A_{-2}\right)$, $\left(A_{3}, A_{3-1}\right)$ or $\left(A_{4}, A_{4-1}\right)$ are below the particular threshold then data can be routing through the path with maximum energy and closer to the sink. If there is a problem with the aggregator node closer to the sink, parallel aggregator will be activated immediately and data will be routed to the sink through that aggregator.

This particular fact is widely ignored in $[2,3]$; therefore the proposed approach not only deals with the energyefficient path selection but also enhances the fault tolerance among sensor nodes $s_{1}, s_{2}, \ldots, s_{n}$ when disseminating datasets of interest to the sink nodes $\sin \mathrm{k}_{-1}, \operatorname{sink}_{-2}, \ldots, \operatorname{sink}_{-n}$. The actual paths are shown with the solid lines and the candidate paths are shown with the dotted lines. In case of problem with the sensor node data can be routed through the candidate path closer to the neighboring node.

Experimental results discussed in Section 4, clearly show that FT-MPPBR (fault-tolerant multipath priority-based routing) scheme has outperformed the existing PBR and flooding protocols mentioned in $[2,3]$.

Actual simulations were not performed in [2] but a mathematical model is provided to support the hypothesis.

The mathematical model and the proposed FT-MPPBR algorithm are discussed in the next section. The mathematical approach discussed below is very similar to the one discussed in $[2,3]$ for predicting energy consumption required for the path selection for a better throughput.

The sensor network can be described as undirected graph $G(V, E)$, where " $V$ " means the set of all nodes and " $E$ " represents the edge or path required for routing datasets.
Path " $P$ " is defined as the set of all nodes at a certain distance " $D$ " from the Sink $_{-i}$, where $V\left(A_{i}\right), V\left(A_{j}\right)$ are the parallel aggregators for enhancing fault tolerance

$$
P=\left(V s_{1}, V s_{2}, \ldots, V\left(A_{i}\right), V\left(A_{j}\right), V_{\text {sink-i }}\right) .
$$

Therefore, communication cost can be defined as

$$
\text { Cost }-P=\frac{\sum E_{\text {con }}}{\operatorname{Cost}\left(D_{i, j}\right)}
$$

which represents the distance between two sensor nodes and "Econ" is the energy consumption required for routing a data to the nearest sink.

$$
\text { If } E_{\mathrm{con}}=0 \text {, }
$$

it means the path is broken alternative path will be selected to ensure network can still provide the desired functionality in the presence of fault.

Fault tolerance in wireless sensor network is crucial to avoid degradation of the whole network. In priority-based routing, packet can be sent to the nearest sink by determining the energy level and routing distance from the nearest sink; main goal is to provide balance between energy level and a routing scheme for prolonging the battery and network lifetime.

As discussed in [2], priority can be calculated as follows:

$$
P_{i, j}=\left(\max \left(\frac{\operatorname{cost}\left(v_{i}, v_{k}\right)}{\operatorname{Cost}\left(v_{i}, v_{k}\right)}\right)\right) A *\left(E_{\mathrm{con}}\right) .
$$

The values of $A, B$ can be set to 1 and zero for calculating the minimum energy cost for routing datasets to the sink node, where " $E_{\text {con }}$ " is the energy consumption level when routing datasets.

3.3. FT-MPPBR Algorithm. FT-MPPBR considers energy consumption and the cost of routing datasets to the sink node but fault tolerance parameter needs to be adjusted with respect to the gradient value. The algorithm mentioned in $[2,3]$ is extended to support fault detection prior to routing datasets of interest.

\section{FT-MPPBR Algorithm. Begin:}

(1) Input: node $P$ with the routing table (RT)

(2) Determine the cost of routing through a certain path to the nearest $\operatorname{sink}_{i}$

(3) if the fault-tolerance (FT) $\geq \operatorname{Max}$ (cost)

(4) then $\operatorname{cost}\left(d_{(m, n)}\right)=E d_{(m, n)}+Z+\mathrm{FT}$, where FT is the fault-tolerance parameter, " $d_{(m, n)}$ " is the distance between two sensor nodes, $E$ is the energy dissipation and $Z$ is the total energy consumption from path " $P$ ".

(5) If path Energy level $(E)=\operatorname{Min}(\mathrm{EL})+\mathrm{FT}$

(6) Select the Candidate path (CP)

(7) IF the Energy level is maximum and faulty Aggregator node $(A i)$ then update the RT 


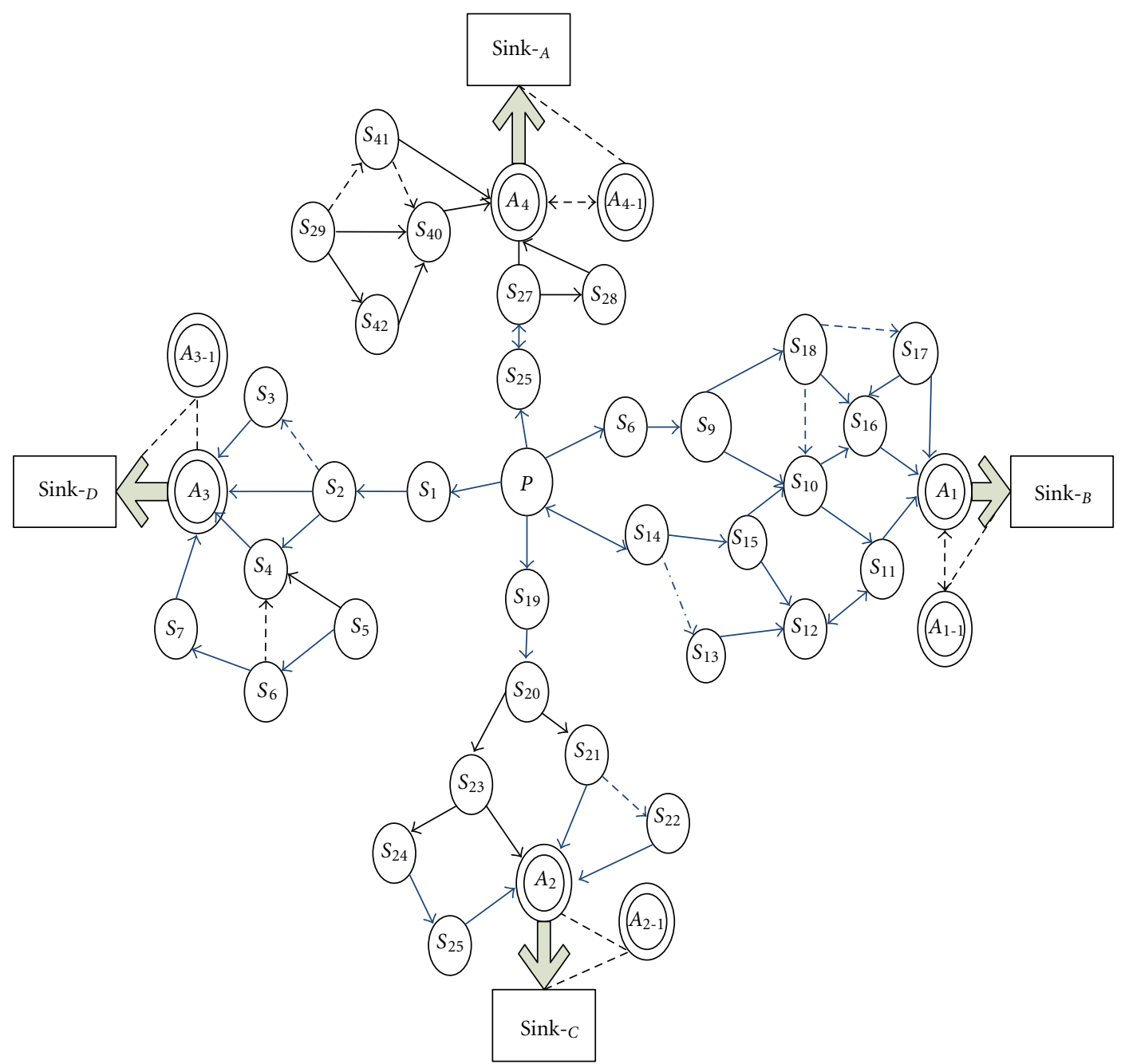

FIGURE 1: Fault-tolerant priority-based routing and aggregation scheme.

(8) temp $=A i$, assign the datasets to $A j$ (parallel aggregator)

(9) If dataset $(\mathrm{ds})=$ Previous $($ dataset $(\mathrm{dp}))$

(10) drop (ds) Else

(11) Aggregator $(A j)=$ Temp,

(12) if temp > max_Priority and

(13) $\operatorname{cost}(d(m, n))=$ Max_energy_Level (ME)

(14) Return the Sink_ID $(s)$

(15) End

FT-MPPBR is a fault tolerant priority-based routing algorithm, which not only takes the energy level into account but also considers malfunctioning before routing datasets of interest to the nearest sink. Our research also looks at the duplicate sensitive datasets efficiently before routing it to the nearest sink, which ensures that similar packets are dropped for reducing communication overheads.

The proposed algorithm is different from $[2,3]$ because it takes into account other parameters such as fault tolerance and duplicate sensitivity for prolonging the life time of healthcare sensor networks.

Healthcare networks are sensitive in many respects such as the following.

(1) Timely dissemination of information is crucial for the survival of a patient

(2) Duplicate sensitivity of datasets depend on the threshold values; if threshold has already been communicated previously then such datasets will be dropped to reduce the network overheads.

(3) Multisinks are used for guaranteeing that the content- and context-sensitive information will be communicated to the relevant GP, soon after the datasets of interest are available.

(4) Computational intensive queries will be processed once the data is communicated to the Grid-sensor gateway. The architecture mentioned in [2] is extended to support grid computing so that datasets received at the nearest sink can be used for future forecasting and well-being of patients. 


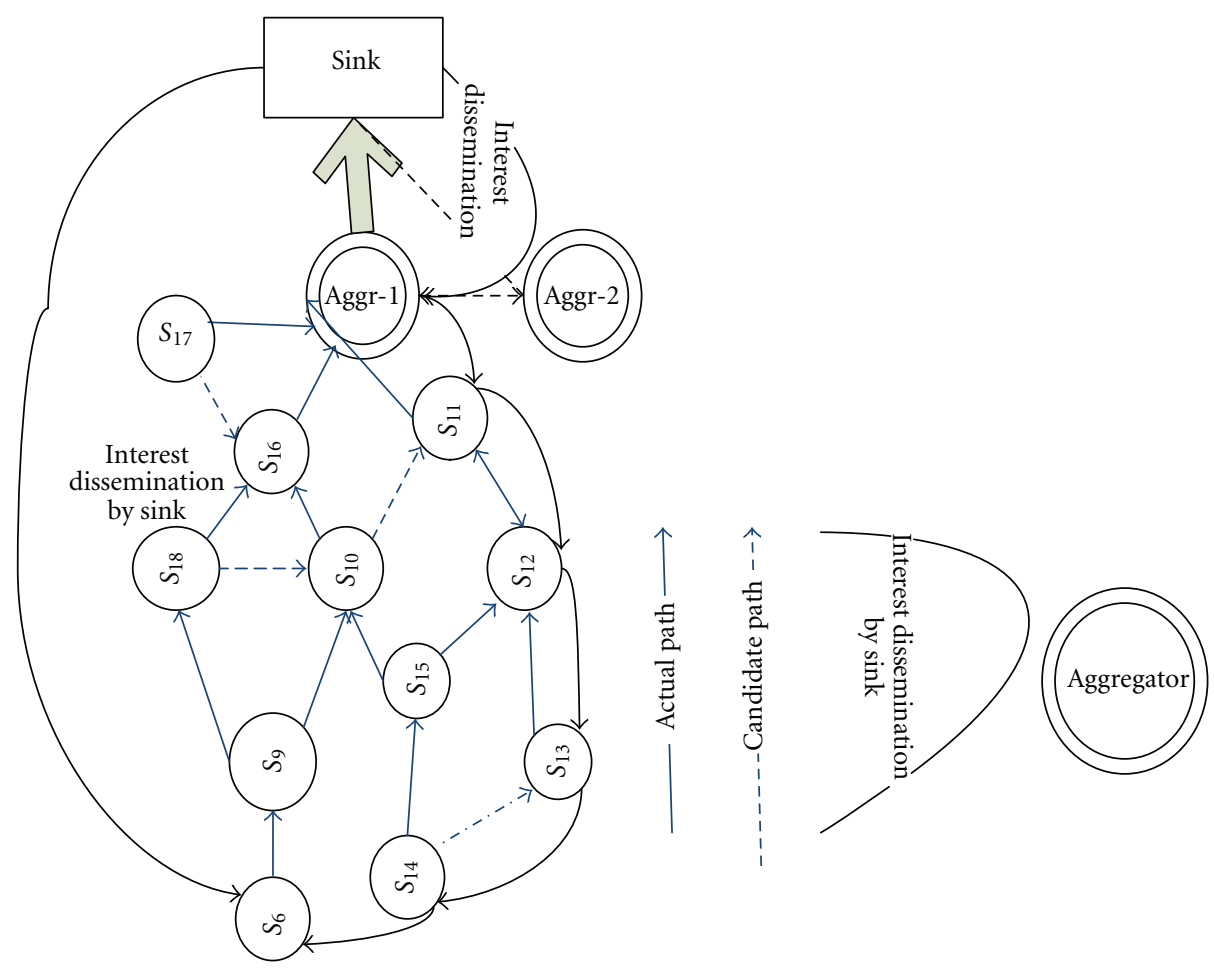

FIGURE 2: Elaboration of the terminologies for multisink path.

The architecture to support the computational intensive queries is shown in Figure 3, which shows transfer of datasets to the sink that is connected to the Grid manager, responsible for the execution of tasks on the remote machines for the fast and efficient processing of received data.

3.4. Fault-Tolerant Multisink Routing Scheme. Priority based multipath routing scheme is shown in Figure 1. The main purpose is to ensure that data can be routed through the candidate or alternative path if there is a problem with the node responsible for answering a particular query. This research focuses on enhancing fault tolerance so that desired functionality can be achieved despite the presence of fault at a particular node close to the sensor network deployment range.

$S_{1}, S_{2}, S_{3}, \ldots, S_{n}$ represent the sensor nodes and Sink $_{-A}$, Sink $-B$, Sink ${ }_{-}$, and Sink - $_{D}$ represent the sinks at different locations. " $P$ " is the priority of the node that will be set once the fault-tolerance parameters are adjusted by looking at the energy consumption constraints.

In the above scheme, path will be established once the datasets of interest are available for routing to the nearest sink, which will be determined after looking at the energy consumption level and the distance of the node from a particular location from point " $p$."

Candidate paths are shown with dotted lines, which represent the alternative paths in case there is any problem with a sensor node.

The malfunctioning of sensor node is important to be considered while communicating datasets of interest to the nearest sink. A single sink does not provide an ideal solution when sensor devices are attached to the patients with serious medical conditions.

The proposed algorithm balances the energy consumption before routing the datasets of interest to the nearest sink by efficiently monitoring malfunctioning of a node responsible for dissemination of datasets.

The parameters which are important to be taken into account such as distance of node " $d$ " from the other sensor nodes, suppose it is $R_{\text {-hop }}$ away from the sink.

Within the $R_{\text {-hop }}$ radius, there could be malfunctioning nodes responsible for answering a particular query or the node might have the information of interest. Such information of interest can be exploited through greedy or diffusion algorithms such as directed diffusion [5], where the query can be forwarded to the next neighbor node with a similar probability as discussed in [3].

The total number of transactions required to find information of interest from sink to the source at a distance ${ }_{(r-1)}$ hop can be calculated as shown below. The proposed approach is very similar to the model discussed in [4], " $N_{i}$ " is the node with relevant information

$$
\text { Transmission }(T)=1+\left[\frac{d}{r}\right](1+N i+r)_{r^{2}}+d
$$

The number of transmissions back to the sink at a distance " $d$ " from the source would be equal to the equation shown 


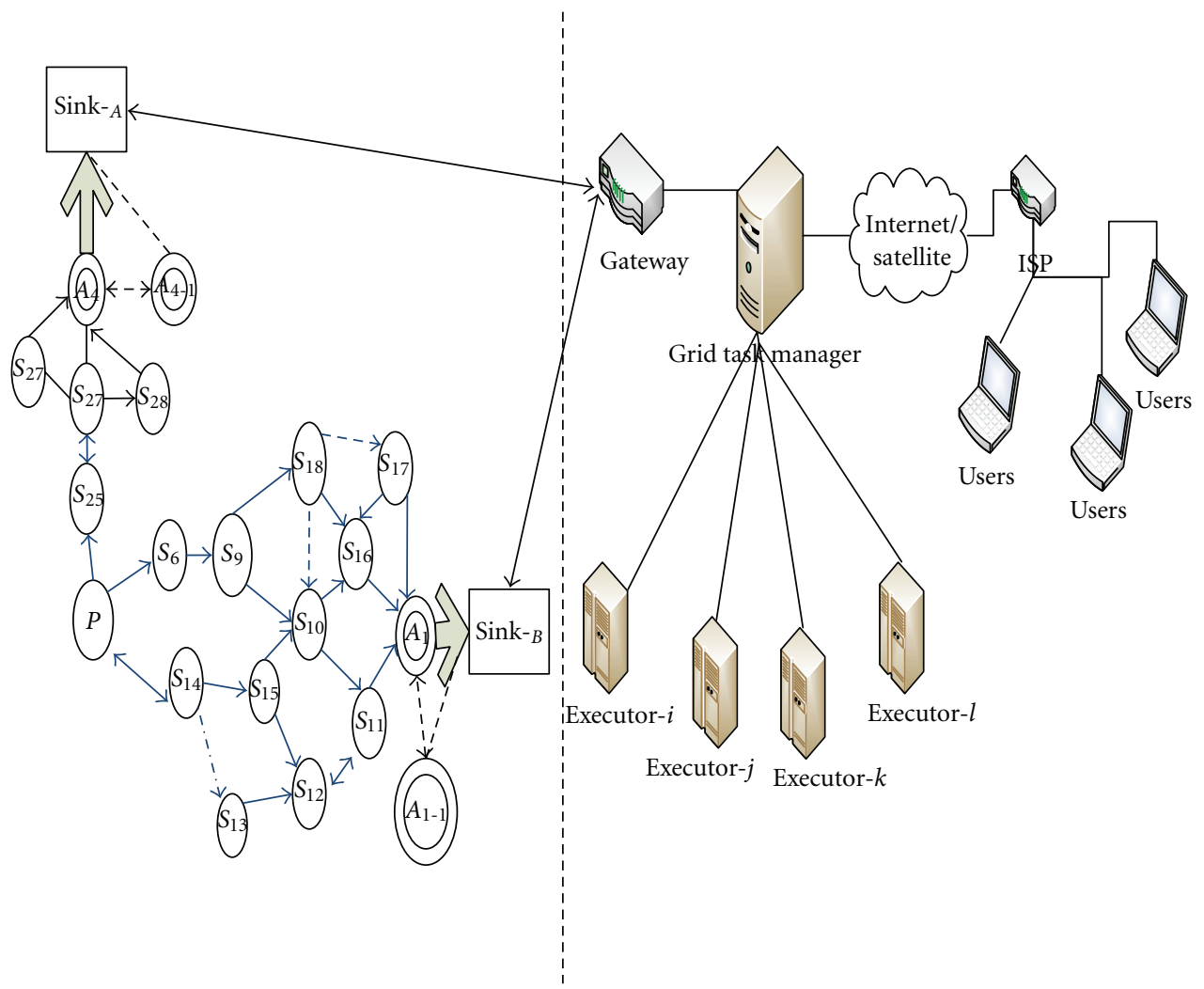

FIgURE 3: Grid-based Multisink architecture.

below, where $\mathrm{Ni}$ is the node with an answer to the relevant query.

$$
\begin{aligned}
\text { Total-Transmission }(T t)= & \left(1+r^{2} N i+r\right)+\left(\left[\frac{d}{r}\right]-1\right) \\
& {\left[1+2 r^{2} N i+r\right]+d . }
\end{aligned}
$$

Therefore, probability of success for the node at the critical path would be equal to the following equation shown below, which is somewhat similar to the equation mentioned in [3], where $\mathrm{Ni}$ is the arbitrary node with the relevant information. Value of the malfunctioning node would be between zero to $R(\max )$ and $P f / 2$ would be the probability of the malfunctioning node within that particular path.

$$
\begin{aligned}
\text { Probability }(P(N i))=\prod_{i=1}^{[d / r]}\lceil 1 & -\frac{P f}{2} \cdot R(\max ) \\
& \left.-f\left(\left(\left[\frac{r}{r}\right]-i\right) r\right]\right] .
\end{aligned}
$$

3.5. Grid-Based Multisink Architecture. The architecture to support computational intensive datasets that are transmitted to the grid-sensor gateway for future forecasting is shown in Figure 3.

The information of interest will be communicated through gateway connected to the $\operatorname{Sin} k_{-A}, \operatorname{Sin} k_{-B}, \ldots, \operatorname{Sin} k_{-n}$.
Computational and resource-intensive tasks will be transferred Executor $_{-1}$, Executor $_{-2}, \ldots$, Executor $-n$ via the grid manager as shown in Figure 3. Finally it will be transferred to the relevant GP via Internet so that issues or problems can be rectified as soon as possible.

The main purpose is to perform early diagnosis of patients with the life-threatening conditions. Fault tolerance is particularly important while dealing with such a sensitive domain for minimizing delays as much as possible for the timely dissemination of information.

Simulation results are encouraging and will be discussed in the next section.

\section{Experimental Analyses}

Extensive simulations are conducted to validate the proposed approach. The objective is to compare the performance of existing routing schemes with the FT-MPPBR algorithm, discussed in Section 3.3.

The performance metrics that need to be considered are the following.

(1) Communication overheads will be increased significantly, if the energy consumption is not properly looked at by the routing scheme.

(2) Fault tolerance of the node depends on the percentage of packets successfully routed to the nearest sink during the presence of a malfunctioning node. Therefore balancing the energy consumption, when 
Comparison of flooding, PBR, and FT-MPPBR scheme

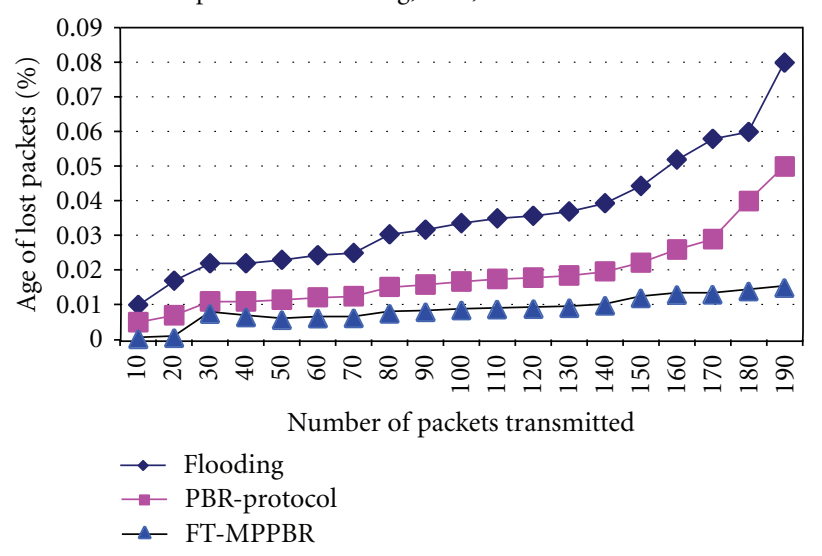

FIGURE 4: Number of packets transmitted.

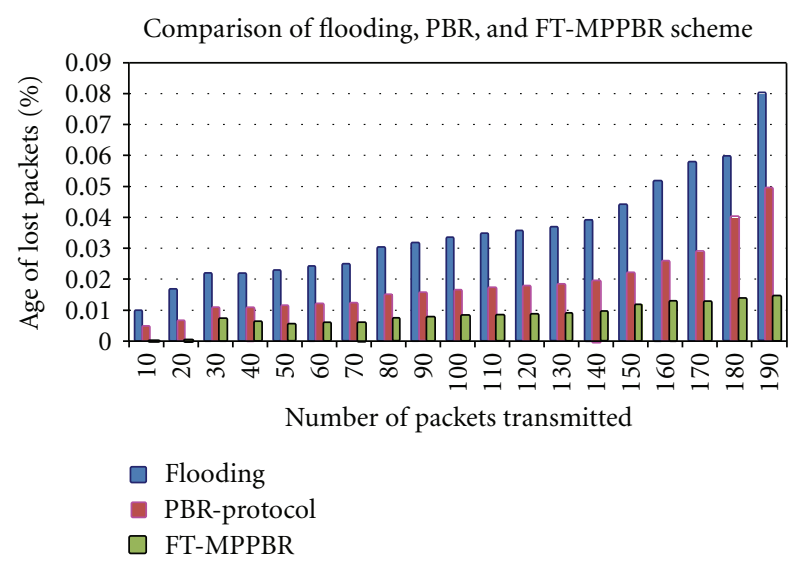

FIGURE 5: Number of packets transmitted.

constructing shortest path to the nearest sink is crucial for achieving fault tolerance.

(3) Distance of the node from nearest sink is an important metric; if the node is further away from the sink, more energy would be consumed when routing datasets and constructing a certain network topology.

(4) Effectiveness of the proposed scheme needs to be measured by comparing it with other priority-based routing schemes.

The simulation results are encouraging and shows that the fault-tolerant priority-based multisink routing scheme (FT-MPPBR) has outperformed in terms of reliability while disseminating datasets to the nearest sink. Simulation results are discussed in the performance evaluation section.

4.1. Simulation and Performance Evaluation. Fault tolerance is particularly important in our simulation model because of the sensitive nature of the healthcare domain. Loss of data packets could be life threatening for patients with serious medical conditions.

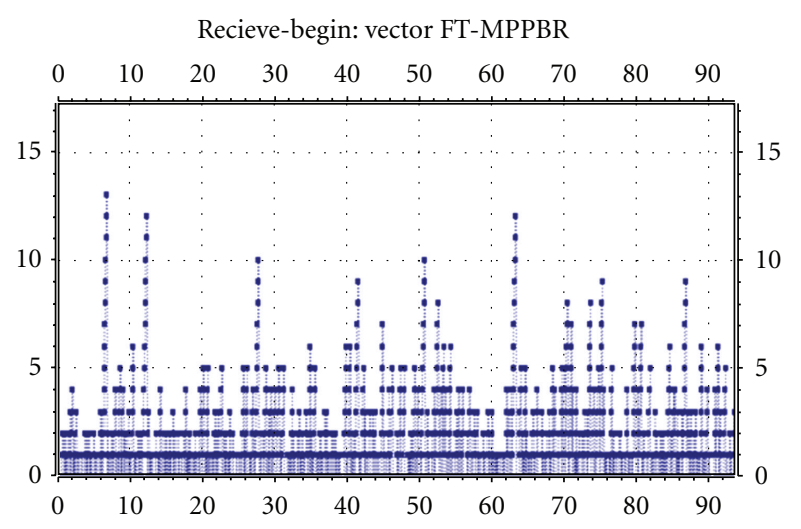

FIgURE 6: Data received and network density.

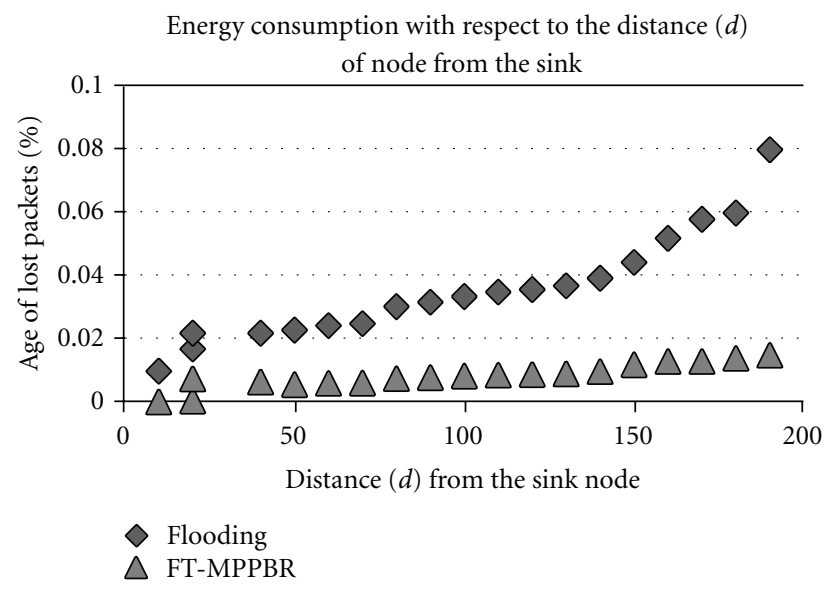

Figure 7: Distance $(d)$ from the sink node.

Figures 4 and 5 typically show the comparison of our proposed FT-MPPBR (fault-tolerant multipath priority-based routing) scheme with the flooding and PBR (priority-based routing) approach for the dissemination of information. The curve in flooding approach is highly skewed and the data loss percentage is also above the normal threshold when compared to other priority-based routing schemes.

Similarly, priority-based routing (PBR) scheme performed better than the flooding approach, but Figures 4 and 5 clearly show that the energy consumption of the PBR approach is still very high when routing datasets.

It is also observed that the proposed FT-MPPBR approach has clearly outperformed the other two approaches in term of balancing energy consumption when routing packets to the nearest sink. Network overheads are also reduced significantly; only those datasets are routed that are crucial and redundant packets were dropped for prolonging the network lifetime.

In the proposed FT-MPPBR approach two things were taken into account, first is the use of parallel aggregator for ensuring fault tolerance and the multipath scheme for routing packets to the nearest sink. It is also observed that 


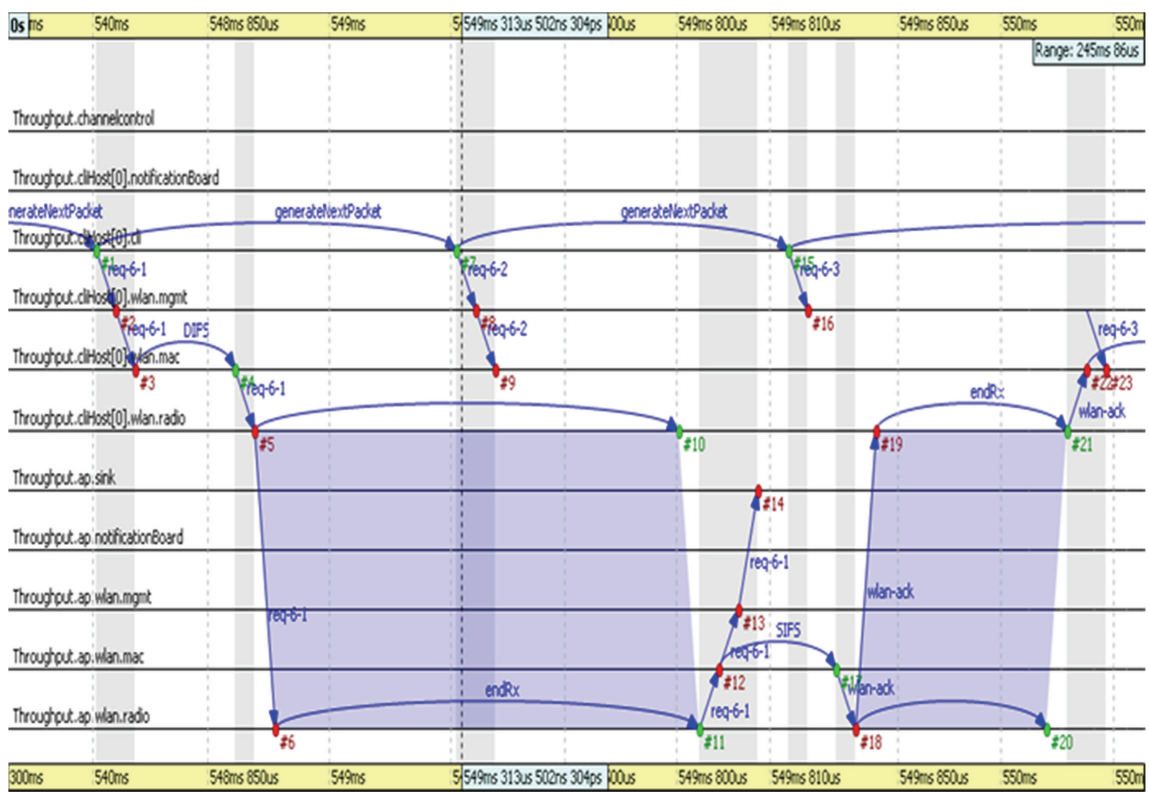

FIgURE 8: Sequence chart 1 of events.

the FT-MPPBR scheme has significantly minimized the data when disseminating datasets of interest.

In other words Figures 4 and 5 graphs clearly show that the data packets received and lost due to collision in FTMPPBR are significantly less as compared to the flooding and PBR (priority-based routing) scheme.

The FT-MPPBR has outperformed in terms of energy consumption for a better throughput. In PBR and flooding, the curve is skewed and the latency is higher when the distance of the packet to send is greater while disseminating datasets of interest to the sink.

Figure 6 shows the data transmission phase and early stages of network density vector in terms of receiving data packets.

The Figure 7 shows that the energy consumption level of FT-MPPBR scheme is significantly low as compared to the PBR scheme for routing datasets. In FT-MPPBR, data aggregation scheme looks at the duplicate sensitive datasets.

Our scheme also has some similarity with directed diffusion [5] when removing duplicate sensitive datasets. We have also observed that the greater the distance of the node from nearest sink, the higher the network latency and the battery energy consumpgor network lifetime.

Figures 8, 9 and 10 show the sequence chart depicting events and datasets that are routed in a particular time to the nearest sink. Blue arrow in the sequence chart represents the message sent through a particular node. The red dot represents the message processing event and green dot represents the processing of data on a particular node at a particular time. The main benefit of using sequence chart is to keep track of the event logs for linear and nonlinear analysis. Omnet++ [36] simulator was used for tracking the sequence of events in wireless sensor networks.

\section{Conclusion}

This paper typically focuses on the detailed analysis and performance comparison of the proposed fault-tolerant multipath priority-based routing scheme (FT-MPPBR) scheme. Fault tolerance has been widely ignored in the existing research literatures, while disseminating datasets of interest to the sink.

We have performed extensive experiments and observed that more energy is consumed in the single sink environment as compared to the multisink PBR schemes. Similarly, single path can easily compromise the quality of sensor networks because of the distance of the node from the sink. Sensor nodes responsible for answering a query might be at a distance far from the sink. Such paths always consume more energy and can result in the degradation of the whole network.

Experimental analysis of the proposed priority-based multipath scheme FT-MPPBR has clearly shown that it has outperformed the existing PBR and flooding approach in terms of efficiently routing datasets to the nearest sink.

The query success rate was dropped significantly in those cases where the network density was high and nodes are at the maximum distance from nearest sink.

Fault tolerance is crucial when wearable sensors are attached to the patients with serious medical conditions. Our problem domain is different from the existing priority-based routing schemes because it includes sensor devices that are attached to patients and malfunctioning of a node could be life threatening.

A parallel aggregator was introduced to achieve desired functionality during the presence of fault. The objective was to ensure that datasets can be routed through different paths with the help of parallel aggregator.

Currently we are further investigating the effectiveness of proposed approach with other multisink and multipath 


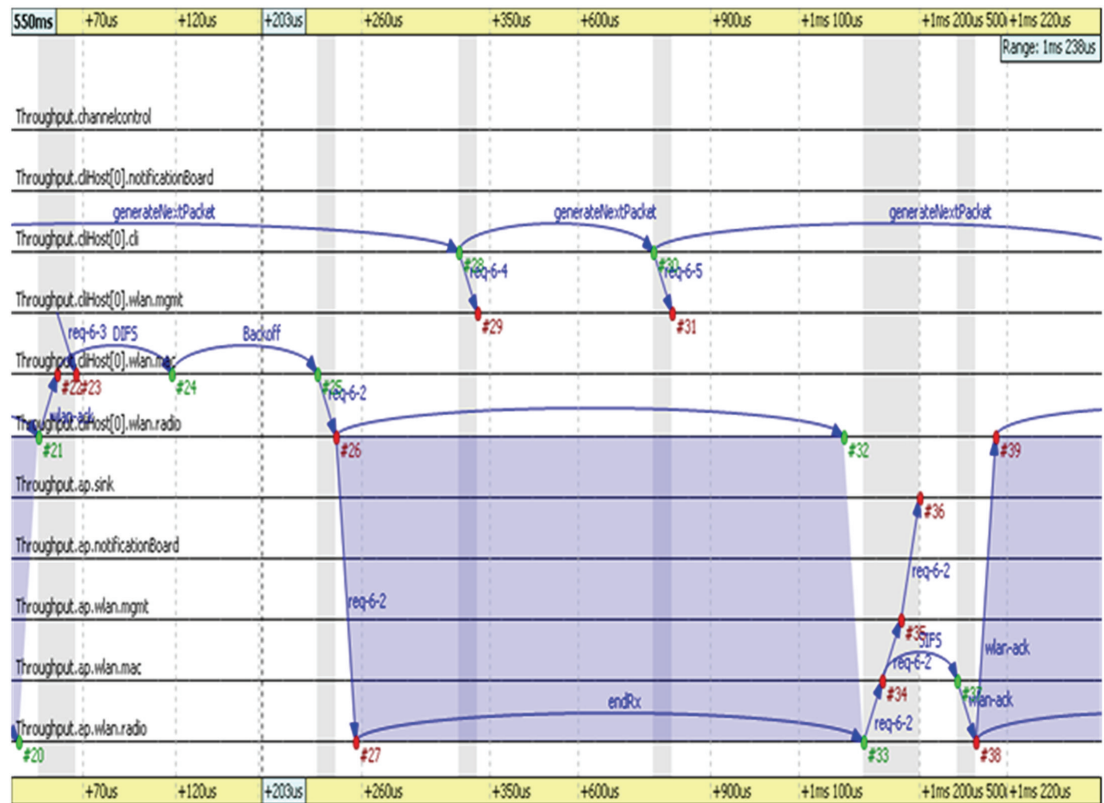

FIgURE 9: Sequence chart 2 of events.

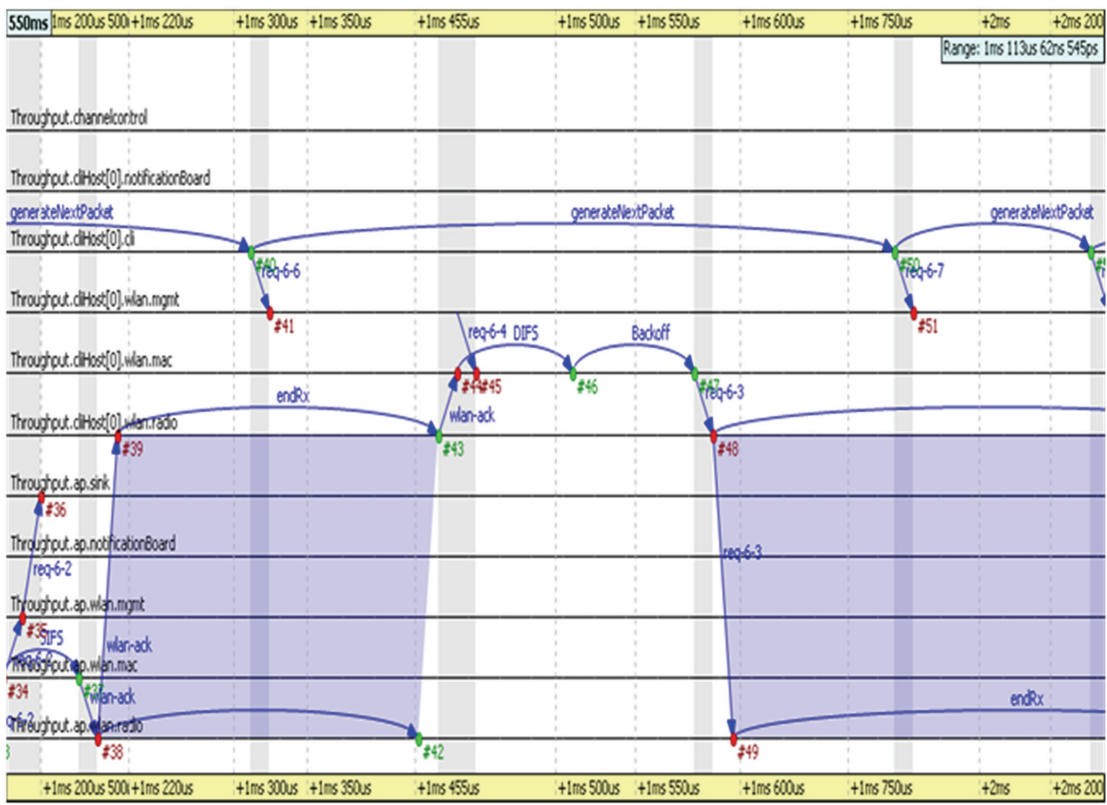

Figure 10: Sequence chart 3 of events.

schemes for further minimizing the network latency and battery energy consumption for a better throughput.

The simulation results are encouraging so far and will be submitted in this Journal or a conference soon.

\section{References}

[1] I. F. Akyildiz, W. Su, Y. Sankarasubramaniam, and E. Cayirci, "A survey on sensor networks," IEEE Communications Magazine, vol. 40, no. 8, pp. 102-114, 2002.

[2] J. Faruque, K. Psounis, and A. Helmy, "Analysis of gradientbased routing protocols in sensor networks," in Proceedings of the IEEE/ACM International Conference on Distributed Computing in Sensor Systems (DCOSS'05), vol. 3560, pp. 258-275, June 2005.

[3] M. Meng, H. Xu, X. Wu et al., "PBR: priority based routing in multi-sink sensor networks," in Proceedings of the International Conference on Wireless Networks (ICWN'07), Los Vegas, Nev, USA, June 2007.

[4] Y. Liu and W.K.G. Seah, "A Priority based routing protocol for sensor networks," in Proceedings of the IEEE 15th International Symposium on Personal, Indoor and Mobile Radio Cmmunications (PIMRC'04), IEEE, Barcelona, Spain, September 2004.

[5] C. Intanagonwiwat, R. Govindan, and D. Estrin, "Directed diffusion: a scalable and robust communication paradigm 
for sensor networks," in Proceedongs of the 6th Annual International Conference on Mobile Computing and Networking (MOBICOM'00), pp. 56-67, ACM, Boston, Mass, USA, August 2000.

[6] F. Koushanfar, M. Potkonjak, and A. Sangiovanni-Vincentelli, "Fault tolerance techniques in wireless ad-hoc sensor networks," UC Berkeley Technical Reports, 2002.

[7] K. Marzullo, "Tolerating failures of continuous valued sensors," ACM Transactions on Computer Systems, vol. 8, no. 4, pp. 284-304, 1990.

[8] E. F. Moore and C. E. Shannon, "Reliable circuits using less reliable relays I," Journal of the Franklin Institute, vol. 262, no. 3, pp. 191-208, 1956.

[9] D. N. Jayasimha, "Fault tolerance in multisensor networks," IEEE Transactions on Reliability, vol. 45, no. 2, pp. 308-315, 1996.

[10] H. Liu, Z. L. Zhang, J. Srivastava, and V. Firoiu, "PWave: a multi-source multi-sink anycast routing framework for wireless sensor networks," in Proceeding of the 6th International IFIP-TC6 Networking Conference, pp. 179-190, May 2007.

[11] R. C. Shah and J. M. Rabaey, "Energy aware routing for low energy ad hoc sensor networks," in Proceedings of the IEEE Wireless Communications and Networking Conference (WCNC'02), vol. 1, pp. 350-355, March 2002.

[12] K. Sohrabi, J. Gao, V. Ailawadhi, and G. J. Pottie, "Protocols for self-organization of a wireless sensor network," IEEE Personal Communications, vol. 7, no. 5, pp. 16-27, 2000.

[13] G. J. Pottie and W. J. Kaiser, "Wireless integrated network sensors," Communications of the ACM, vol. 43, no. 5, pp. 51$58,2000$.

[14] Y. Yao and J. Gehrke, "Query processing for sensor networks," in Proceedings of the Conference on Innovative Data Systems Research, Asilomar, Calif, USA, 2003.

[15] Y. C. Tseng, S. Y. Ni, Y. S. Chen, and J. P. Sheu, "The broadcast storm problem in a mobile ad hoc network," Wireless Networks, vol. 8, no. 2-3, pp. 153-167, 2002.

[16] S. Lindsey and C. S. Raghavendra, "PEGASIS: power efficient gathering in sensor information systems," in Proceedings of the IEEE Aerospace Conference, Big Sky, Mont, USA, March 2002.

[17] W. Heinzelman, J. Kulik, and H. Balakrishnan, "Adaptive protocols for information dissemination in wireless sensor networks," in Proceedings of the 5th Annual ACM/IEEE International Conference on Mobile Computing and Networking (MobiCom_99), ACM, Seattle, WA, USA, August 1999.

[18] J. Liu, F. Zhao, and D. Petrovic, "Information-directed routing in ad hoc sensor networks," in Proceedings of the ACM 2nd International Workshop on Wireless Sensor Networks and Applications (WSNA'03), pp. 88-97, September 2003.

[19] M. Youssef, M. Younis, and K. Arisha, "A constrained shortestpath energy-aware routing algorithm for wireless sensor networks," in Proceedings of theIEEE Wireless Communication and Networks Conference (WCNC '02), Orlando, Fla, USA, March 2002.

[20] C. Intanagonwiwat, D. Estrin, R. Govindan, and J. Heidemann, "Impact of network density on data aggregation in wireless sensor networks," in Proceedings of the 22nd International Conference on Distributed Systems (ICDCS'02), pp. 457458, July 2002.

[21] J. Heidemann, F. Silva, and D. Estrin, "Matching data dissemination algorithms to application requirements," in Proceedings of the 1st International Conference on Embedded Networked Sensor Systems (SenSys'03), pp. 218-229, November 2003.

[22] D. Braginsky and D. Estrin, "Rumor routing algorithm for sensor networks," in Proceedings of the ACM 1st Interna- tional Workshop on Wireless Sensor Networks and Applications (WSNA'02), pp. 22-31, September 2002.

[23] L. M. L. de Lau and M. M. B. Breteler, "Epidemiology of Parkinson's disease," The Lancet Neurology, vol. 5, no. 6, pp. 525-535, 2006.

[24] N. L. W. Keijsers, M. W. I. M. Horstink, and S. C. A. M. Gielen, “Ambulatory motor assessment in Parkinson's disease," Movement Disorders, vol. 21, no. 1, pp. 34-44, 2006.

[25] J. I. Hoff, B. J. van Hilten, and R. A. C. Roos, "A review of the assessment of dyskinesias," Movement Disorders, vol. 14, no. 5, pp. 737-743, 1999.

[26] A. J. Manson, P. Brown, J. D. O’Sullivan, P. Asselman, D. Buckwell, and A. J. Lees, "An ambulatory dyskinesia monitor," Journal of Neurology Neurosurgery and Psychiatry, vol. 68, no. 2, pp. 196-201, 2000.

[27] R. J. Dunnewold, J. I. Hoff, H. C. van Pelt, P. Q. Fredrikze, E. A. Wagemans, and B. J. van Hilten, "Ambulatory quantitative assessment of body position, bradykinesia, and hypokinesiain parkinson's disease," Journal of Clinical Neurophysiology, vol. 15, no. 3, pp. 235-242, 1998.

[28] A. Salarian, H. Russmann, F. J. G. Vingerhoet et al., "An ambulatory system to quantify bradykinesia and tremor in Parkinson's disease," in Proceedings of the 4th International IEEE EMBS Special Topic Conference on Information Technology Applications in Biomedicine, pp. 35-38, April 2003.

[29] A. Salarian, H. Russmann, F. J. G. Vingerhoets et al., "Gait assessment in Parkinson's disease: toward an ambulatory system for long-term monitoring," IEEE Transactions on Biomedical Engineering, vol. 51, no. 8, pp. 1434-1443, 2004.

[30] S. Madden, M. J. Franklin, J. M. Hellerstein, and W. Hong, "TAG: a tiny aggregation service for ad-hoc sensor networks," in Proceedings of the Operating Systems Design and Implementation (OSDI'02), 2002.

[31] C. Intanagonwiwat, D. Estrin, R. Govindan, and J. Heidemann, "Impact of network density on data aggregation in wireless sensor networks," in Proceedings of the 22nd International Conference on Distributed Systems (CDCS'02), pp. 457458, July 2002.

[32] J. Heidemann, F. Silva, and D. Estrin, "Matching data dissemination algorithms to application requirements," in Proceedings of the 1st International Conference on Embedded Networked Sensor Systems (SenSys'03), pp. 218-229, November 2003.

[33] Y. Xu, J. Heidemann, and D. Estrin, "Geography-informed energy conservation for ad hoc routing," in Proceedings of the 7th Annual ACM/IEEE International Conference on Mobile Computing and Networking (MobiCom '01), Rome, Italy, July 2001.

[34] N. Sadagopan, B. Krishnamachari, and A. Helmy, "Active query forwarding in sensor networks (ACQUIRE)," SNPA, 2003.

[35] M. Chu, H. Haussecker, and F. Zhao, "Scalable informationdriven sensor querying and routing for ad hoc heterogeneous sensor networks," International Journal of High Performance Computing Applications, vol. 16, no. 3, pp. 293-313, 2002.

[36] Omnet++ Similulator download, http://www.omnetpp.org/. 

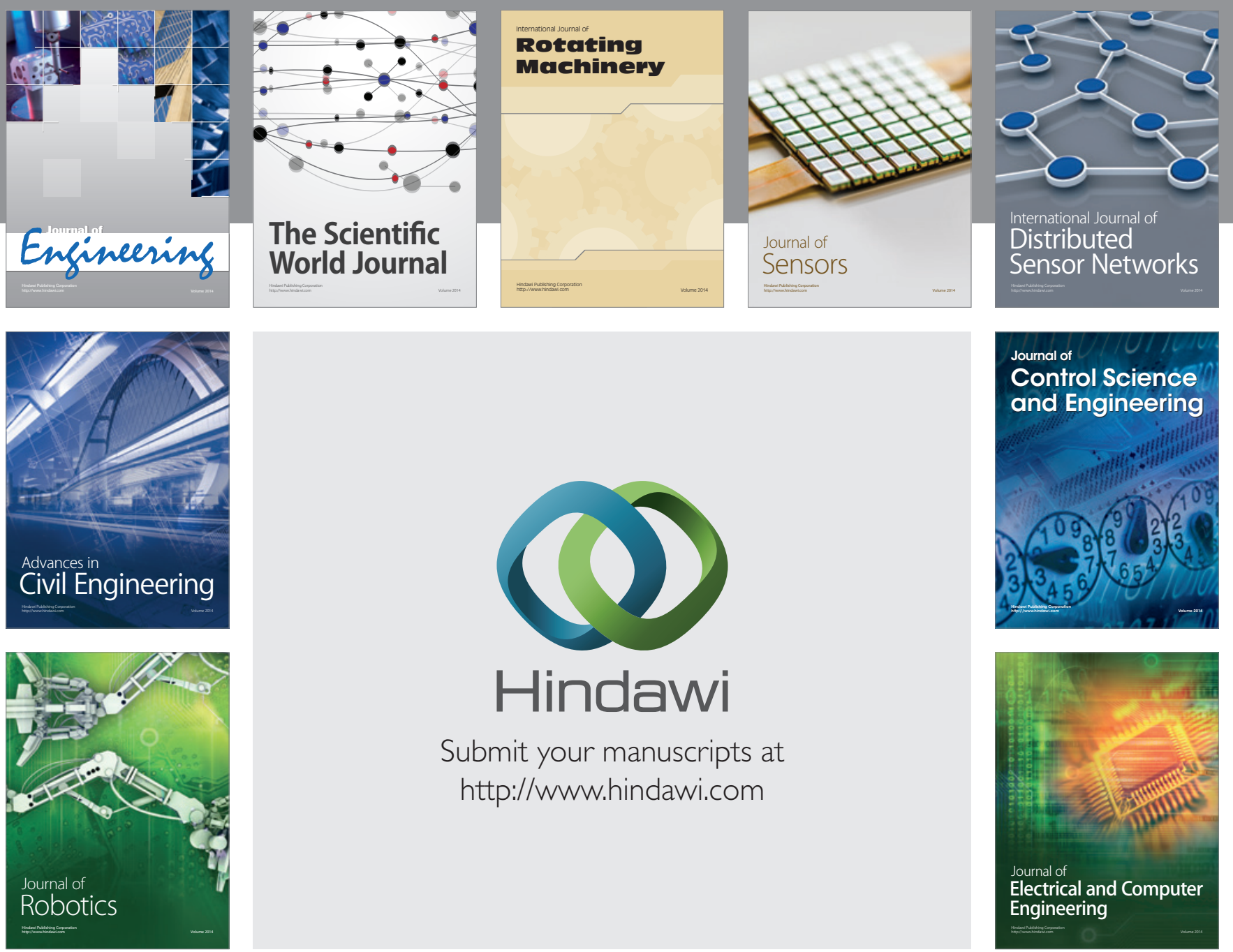

Submit your manuscripts at

http://www.hindawi.com
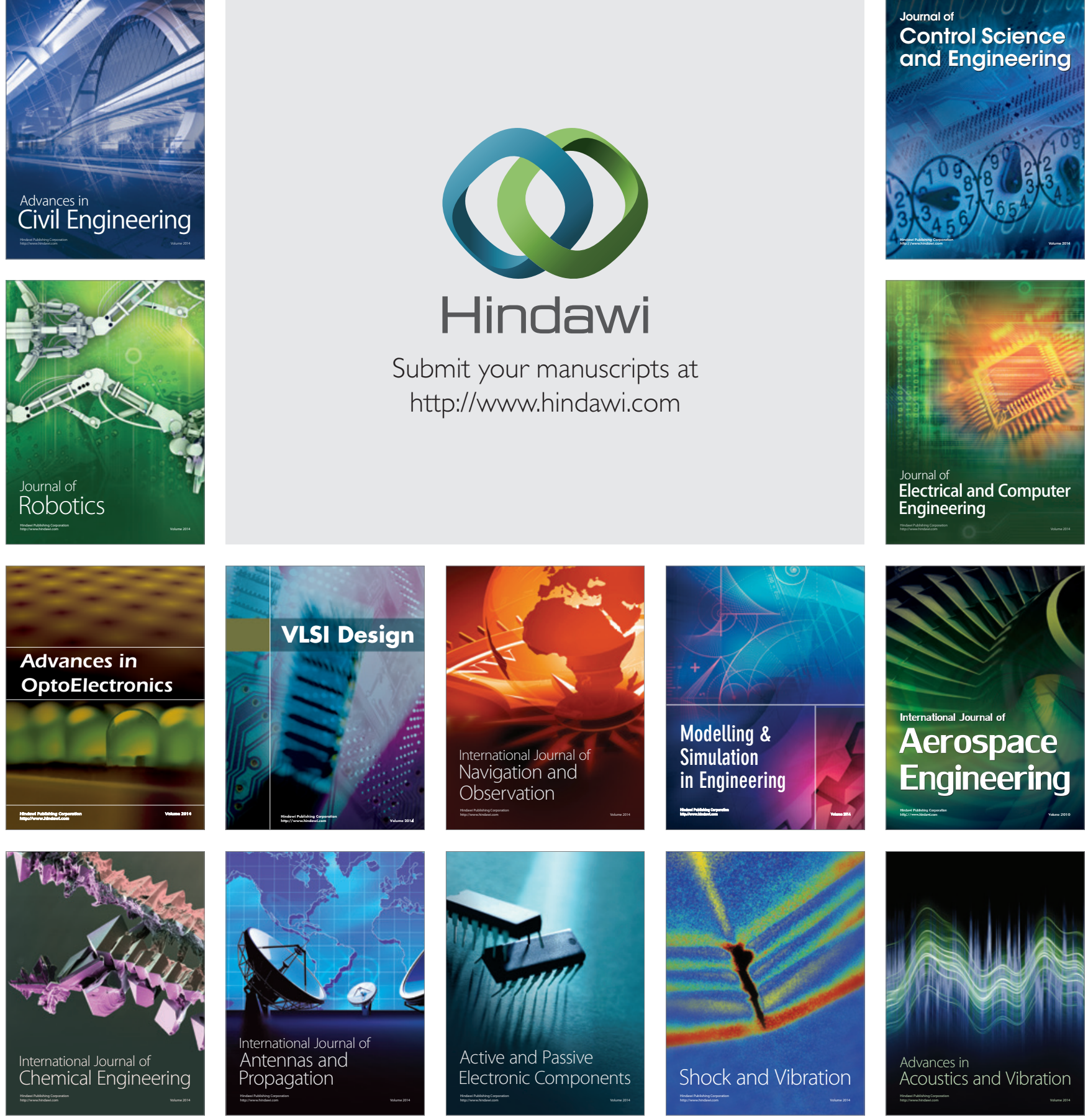